\section{Nontypable Haemophilus influenzae: an intracellular phase within epithelial cells might contribute to persistence}

In this issue, Morey et al. (2011) present experimental evidence showing intracellular survival of nontypable Haemophilus influenzae (NTHi) in infected epithelial cells in vitro. After internalization, bacteria seem to remain in a quiescent non-replicative state inside an acidic subcellular compartment with markers of late endosomes, which the authors name the NTHi-containing vacuole (NTHi-CV). While invasive disease caused by encapsulated type b $H$. influenzae has been efficiently controlled in developed countries due to the widespread use of the conjugated vaccine, unencapsulated NTHi remains prevalent in the aetiology of bacterial respiratory infections, especially in chronic obstructive pulmonary disease (COPD) patients. It is known that NTHi can persistently colonize the respiratory tract of COPD patients, despite culturenegative sputum, and, more importantly, despite the presence of antibodies against the pathogen (Murphy et al., 2004). This strongly suggests that NTHi has found a way to hide in the tissue. The data of Morey and colleagues showing that NTHi can invade airway epithelial cells at low rates and remain in a metabolically active state intracellularly may shed some light on the potential reservoir for persistent NTHi colonizers. Such an intracellular niche would enable bacteria to resist antibiotic therapy and be protected from the adaptive immune response of the host. It remains to be demonstrated whether these bacteria can then exit cells to be released back into the respiratory tract and be associated with the severe exacerbation episodes characteristic of COPD patients. Nonetheless, the possibility that NTHi has a transient intracellular state during infection should now be considered to improve therapy and management of COPD patients chronically infected with NTHi.

The virulence determinants of this opportunistic pathogen are largely unknown. Biofilm formation has been related to the chronicity and severity of the disease, although the involvement of biofilms in pathogenicity is still a matter of controversy (reviewed by Moxon et al., 2008; Erwin \& Smith, 2007). Also, invasive behaviour has been related to the presence of phosphatidylcholine (PCho) in the exposed lipooligosaccharide (LOS) (Swords et al., 2000), which has also been related to biofilm formation and the resistance to clearance of the bacteria in an animal model (Pang et al., 2008; Hong et al., 2007). In this report, Morey and colleagues conclude that invasion of epithelial cells is independent of the presence of PCho in the LOS, but that the process requires membrane lipid rafts, phosphatidylinositol 3-kinase (PI3K) activity and the involvement of tubulin, but not the actin cytoskeleton. This adds new perspectives to our understanding of the strategies of interaction of NTHi with the host.

The study by Morey and colleagues raises new questions that must now be addressed experimentally to establish the clinical relevance of their observations. Encouragingly, the in vitro infection setting established in this report should allow identification in the future of the molecular determinants of invasion and the factors that modulate maturation of the NTHi-CV and their potential arrest in a compartment with late-endosomal features. A note of caution should be added, since several recent studies have highlighted the fact that pathogens formerly thought to avoid fusion with lysosomes (such as Salmonella and Brucella) do in fact interact significantly with these compartments, a process only visible with live cell imaging (Drecktrah et al., 2007; Starr et al., 2008). On a bacterial level, it would be interesting to analyse the switch of virulent extracellular NTHi into a quiescent intracellular state, perhaps by further characterizing the physiological properties of these latent bacteria. Understanding the mechanisms underlying the intracellular behaviour of NTHi might shed some light on the pathology of the disease, as well as on the elusive question of what enables NTHi to persist within patients hidden from the host inflammatory response.

\section{Suzana P. Salcedo ${ }^{1,2,3}$ and Víctor J. Cid ${ }^{4}$}

${ }^{1}$ Aix Marseille Université, Faculté des Sciences de Luminy, Centre d'Immunologie de Marseille-Luminy (CIML), UMR6546, Marseille, F-13288, France

${ }^{2}$ Institut National de la Santé et de la Recherche Médicale (INSERM), U631, F-13288, France

${ }^{3}$ Centre National de la Recherche Scientifique (CNRS), UMR6102, Marseille, F-13288, France

${ }^{4}$ Dpto de Microbiología II, Facultad de Farmacia, Universidad Complutense de Madrid, Instituto Ramón y Cajal de Investigaciones Sanitarias (IRyCIS), Madrid-28040, Spain

\footnotetext{
Microbiology Comment provides a forum for discussion of scientific issues arising directly from papers published in the journal. The authors of papers under discussion will be offered an opportunity to respond.

Guidelines on how to submit a Microbiology Comment article can be found in the Instructions for Authors at http://mic.sgmjournals.org

It should be noted that the Editors of Microbiology do not necessarily agree with the views expressed in Microbiology Comment.

A. Fouet, Editor-in-Chief
} 
Correspondence: Víctor J. Cid

(vicjcid@farm.ucm.es)

Drecktrah, D., Knodler, L. A., Howe, D. \& Steele-Mortimer, O. (2007). Salmonella trafficking is defined by continuous dynamic interactions with the endolysosomal system. Traffic 8, 212-225.

Erwin, A. L. \& Smith, A. L. (2007). Nontypeable Haemophilus influenzae: understanding virulence and commensal behavior. Trends Microbiol 15, 355-362.

Hong, W., Pang, B., West-Barnette, S. \& Swords, W. E. (2007). Phosphorylcholine expression by nontypeable Haemophilus influenzae correlates with maturation of biofilm communities in vitro and in vivo. J Bacteriol $\mathbf{1 8 9}$ 8300-8307.
Morey, P., Cano, V., Marti-Lliteras, P., López-

Gómez, A., Regueiro, V., Saus, C.,

Bengoechea, J. A. \& Garmendia, J. (2011).

Evidence for a non-replicative intracellular stage of nontypable Haemophilus influenzae in epithelial cells. Microbiology 157, 234-250.

Moxon, E. R., Sweetman, W. A., Deadman, M. E., Ferguson, D. J. \& Hood, D. W. (2008).

Haemophilus influenzae biofilms: hypothesis or fact? Trends Microbiol 16, 95-100.

Murphy, T. F., Bruer, A. L., Schiffmacher, A. T. \& Sethi, S. (2004). Persistent colonization by Haemophilus influenzae in chronic obstructive pulmonary disease. Am J Respir Crit Care Med 170, 266-272.

Pang, B., Winn, D., Johnson, R., Hong, W., WestBarnette, S., Kock, N. \& Swords, W. E. (2008).
Lipooligosaccharides containing phosphorylcholine delay pulmonary clearance of nontypeable Haemophilus influenzae. Infect Immun 76, 2037-2043.

Starr, T., Ng, T. W., Wehrly, T. D., Knodler, L. A. \& Celli, J. (2008). Brucella intracellular replication requires trafficking through the late endosomal/lysosomal compartment. Traffic $\mathbf{9}$, 678-694.

Swords, W. E., Buscher, B. A., Ver Steeg, K., II, Preston, A., Nichols, W. A., Weiser, J. N., Gibson, B. W. \& Apicella, M. A. (2000). Nontypeable Haemophilus influenzae adhere to and invade human bronchial epithelial cells via an interaction of lipooligosaccharide with the PAF receptor. Mol Microbiol 37, 13-27.

DOI 10.1099/mic.0.046722-0 